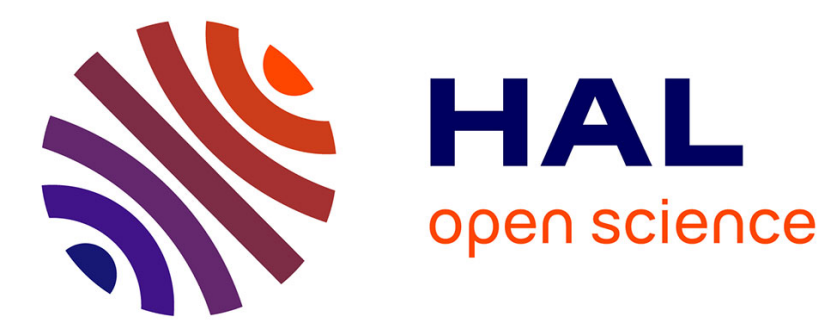

\title{
Calling Behavior and Sex Pheromone Release and Storage in the Moth Chloridea virescens
}

\author{
Stephen P Foster, Karin G Anderson, Jérome Casas
}

\section{To cite this version:}

Stephen P Foster, Karin G Anderson, Jérome Casas. Calling Behavior and Sex Pheromone Release and Storage in the Moth Chloridea virescens. Journal of Chemical Ecology, 2020, 46 (1), pp.10-20. 10.1007/s10886-019-01133-w . hal-02573248

\section{HAL Id: hal-02573248 \\ https://hal.science/hal-02573248}

Submitted on 14 May 2020

HAL is a multi-disciplinary open access archive for the deposit and dissemination of scientific research documents, whether they are published or not. The documents may come from teaching and research institutions in France or abroad, or from public or private research centers.
L'archive ouverte pluridisciplinaire HAL, est destinée au dépôt et à la diffusion de documents scientifiques de niveau recherche, publiés ou non, émanant des établissements d'enseignement et de recherche français ou étrangers, des laboratoires publics ou privés. 
Address correspondence to: Dr. Stephen Foster North Dakota State University Entomology Department NDSU Dept 7650

PO Box 6050

Fargo, ND 58108-6050

U.S.A

Ph. 1-701-231-6444

Fax 1-701-231-8557

Email: stephen.foster@ndsu.edu

\title{
Calling behavior and sex pheromone release and storage in the moth Chloridea virescens
}

\author{
Stephen P. Foster ${ }^{1}$, Karin G. Anderson ${ }^{1}$ and Jérôme Casas $^{2}$ \\ ${ }^{1}$ Entomology Department, North Dakota State University, \\ PO Box 6050, Fargo, North Dakota 58108-6050, U.S.A \\ and \\ 2Institut de Recherche sur la Biologie de l'Insecte, \\ IRBI-UMR CNRS 7261, Université de Tours, 37200 Tours, FRANCE
}

Keywords: Pheromone gland, mass isotopomer distribution analysis, Lepidoptera, biosynthesis, catabolism, titer 


\section{Abstract}

Female moths release sex pheromone to attract mates. In most species, sex pheromone is produced in, and released from, a specific gland. In a previous study, we used empirical data and compartmental modeling to account for the major pheromone gland processes of female Chloridea virescens: synthesis, storage, catabolism and release. Surprisingly, we found that females released little (20-30\%) of their pheromone, with most catabolized. The recent publication of a new pheromone collection method led us to reinvestigate pheromone release and catabolism in $C$. virescens on the basis that our original study might have underestimated release rate (thereby overestimating catabolism) due to methodology and females not calling (releasing) continuously. Further we wished to compare pheromone storage/catabolism between calling and non-calling females. First, we observed calling intermittency of females. Then, using decapitated females, we used the new collection method, along with compartmental modeling, gland sampling and stable isotope labeling, to determine differences in pheromone release, catabolism and storage between (forced) simulated calling and non-calling females. We found, (i) intact $1 \mathrm{~d}$ females call intermittently; (ii) pheromone is released at a higher rate than previously determined, with simulations estimating that continuously calling females release ca. $70 \%$ of their pheromone (only $30 \%$ catabolized); (iii) extension (calling)/retraction of the ovipositor is a highly effective “on/off' mechanism for release; (iv) both calling and non-calling females store most pheromone on or near the gland surface, but calling females catabolize less pheromone; (v) females are capable of producing and releasing pheromone very 
rapidly. Thus, not only is the moth pheromone gland efficient, in terms of the proportion of pheromone released Vs. catabolized, but it is highly effective at shutting on/off a high flux of pheromone for release. 


\section{INTRODUCTION}

The release and perception of sex pheromone is critical for mating in most species of moths (Allison and Cardé 2016a). Typically, the female of a species produces sex pheromone and releases it from a gland located on the intersegmental membrane between the $8^{\text {th }}$ and $9^{\text {th }}$ abdominal segments (Ma and Ramaswamy 2003). Female moths usually produce and release pheromone over a defined period of the day (Groot 2014), or until mating occurs (Foster 1993). Release is usually characterized by a behavior termed "calling", in which a female extrudes the ovipositor to expose and evert the gland, allowing release of the semivolatile compound(s) (Allison and Cardé 2016b; Bendib and Minet 1998). It is thought that extrusion/retraction of the gland allows a female to start/stop release of pheromone, although this has rarely, if ever, been tested (but see Nojima et al. 2018). Female moths may call continuously or intermittently throughout their sexually active period (Allison and Cardé 2016b).

Recently, we modeled empirical data to explore the relationship among pheromone production, storage (titer) and release in the moth Chloridea (formerly Heliothis) virescens Fabricius (family Noctuidae). The most surprising result was the small percentage (20-30\%) of pheromone produced that was actually released, with the balance catabolized to unknown products (Foster et al. 2018). Given the critical role of sex pheromone in mating, this high level of "loss" (i.e., not used in signaling) seemed counter to the idea that pheromone is efficient and costly to produce (Johansson and Jones 2007). It seemed perplexing to us that females would make relatively small quantities of pheromone, yet catabolize so much. 
Around the time we published our model, Nojima et al. (2018) published a simple and efficient method for collecting pheromone from calling Heliothis subflexa females, in which a small funnel connected to a vacuum pump was placed near the pheromone gland of a calling female to collect pheromone in a small section of coated fused silica column. This method yielded pheromone release rates substantially higher than previous studies using the common method (which we used in our study) of solid adsorbent capture of pheromone released by females in containers (Nojima et al. 2018). This obtained higher release rate could be attributed to three possible improvements: (i) a much more direct path of pheromone to adsorbent, reducing loss in the apparatus (e.g., adsorption on glass), (ii) more efficient adsorption/desorption on the fused silica coating, and (iii) only collecting when females were actually calling; when females stopped calling, the vacuum pump was switched off and not turned on again until females called again (Nojima et al. 2018). We surmised that this method might yield a higher pheromone release rate (and hence lower amount catabolized) for $C$. virescens females, especially as, in our study, we collected for a set time and did not observe directly whether females called throughout. Noctuid moths, in particular, are known to call intermittently and somewhat unpredictably (Almeida et al. 2008; Turgeon and McNeil 1983).

In this study, we had three aims: (i) determine whether female $C$. virescens called continuously or intermittently, (ii) reinvestigate pheromone release rate using a 
modified version of the method of Nojima et al. (2018), and (iii) determine how the behavior of calling influences storage, catabolism and release of pheromone. Specifically, we addressed five questions:

1) What is the duration and intermittency of calling through the sexually active period (scotophase)?

2) Do females release pheromone at a higher rate than originally determined and, if so, how does this affect the amount catabolized?

3) Is calling behavior an effective on/off method for pheromone release?

4) How does storage and catabolism of pheromone differ between calling and non-calling females?

5) Is there a relatively high pheromone flux from production to release?

\section{Methods and Materials}

Insects

Our colony of $C$. virescens originated from a colony reared at the USDA-ARS, Fargo, ND, supplemented with insects from a colony at Department of Entomology, North Carolina State University (kindly supplied by Dr. F. Gould). Larvae were reared on a wheatgerm-casein diet, and the two sexes separated after pupation. Newly eclosed adult females were collected each day and maintained at $25^{\circ} \mathrm{C}$ under a 16:8 L:D photoperiod, without access to liquid (to facilitate later ingestion of labeled

glucose). Most females eclosed during the scotophase and were used in the following scotophase (i.e., nominally $1 \mathrm{~d}$ old). 


\section{Pheromone sampling}

Using intact females presented a problem in that we could not control calling over time, thus precluding a rigorous comparison of pheromone between calling and non-calling females. Consequently, we decided to use decapitated females, which do not call (Foster et al. 2018), and forced them to evert the gland by placing a small vascular clamp (10 x 2.15 mm, Fine Science Tools Inc., Foster City, CA) across the posterior of the abdomen. Thus, we could compare quantities (released and in the gland) between "simulated calling" and "non-calling" females. This approach had a further consequence. Decapitated females do not produce pheromone because the supply of pheromone biosynthesis-activating neuropeptide (PBAN), which stimulates biosynthesis in the gland (Eltahlawy et al. 2007; Jurenka 2017) is cut off. This effectively allowed us to control the amount of pheromone in the gland at the start of an experiment and follow its fate over time (see analyses below), without complication of more pheromone being introduced into the system. We did not test this approach with normal females, as we were concerned that the clamp would cut off PBAN circulation to the gland. The limitation in this approach, however, is that the amounts of pheromone quantified are likely to be less than that produced (and released) by a normal female, which would keep producing pheromone over the course of an experiment.

i) Whole gland extract We sampled total pheromone in and on the surface of the gland by excising it and placed it in $n$-heptane, along with 50 ng of internal standard [IS; (Z)-11-tetradecenal, Pherobank, Wageningen, The Netherlands]. The gland was 
allowed to extract for at least $1 \mathrm{~h}$ at ambient temperature before injection of the extract into the gas chromatograph/mass spectrometer (GC/MS).

ii) Rinse/Extract This obtains two samples of pheromone from a gland: a rinse of the cuticular surface (and possibly deeper) and an extract sampling deeper into the cuticle and into gland cells (Foster and Anderson 2018). Briefly, an extruded gland was rinsed with ca. $50 \mu \mathrm{l}$ of $n$-heptane using a gastight syringe, and the rinse collected in a glass tube with $50 \mathrm{ng}$ of IS. Excess solvent on the gland was blotted with filter paper (Whatman \#5), to limit carryover to the subsequent extract, and the paper placed in the tube along with the rinse. The gland was then excised, placed in $n$-heptane with $50 \mathrm{ng}$ IS and allowed to extract for at least $1 \mathrm{~h}$ at ambient temperature. Both samples were analyzed by GC/MS.

iii) Collection According to the method of Nojima et al. (2018), we fashioned a small glass funnel out of a Pasteur pipette and attached this to a $200 \mathrm{~mm}$ section of a DB1coated fused silica column (530 $\mu \mathrm{m}$ i.d., $5 \mu \mathrm{m}$ film thickness; Agilent Technologies, Santa Clara, CA). A pump (KNF Neuberger, Trenton, NJ, model NMS010S) pulled unfiltered air through the column at an estimated 3-5 ml.min ${ }^{-1}$. We used two types of funnel (Fig. 1): one with a narrow opening (ca. $2.2 \mathrm{~mm}$ i.d.), which fitted around the gland, and the other with a wider opening (ca. $5.8 \mathrm{~mm}$ i.d.), which fitted around the abdomen (Fig 1). The respective funnels were fitted over the gland or abdomen, and pheromone collected for 30 or $60 \mathrm{~min}$., after which the funnel and column were rinsed with ca. $100 \mu \mathrm{l}$ of $n$-hexane and $50 \mathrm{ng}$ of IS, and the eluate collected and 
concentrated before injection into the GC/MS. Blank runs of the system showed undetectable levels of ambient pheromone in the laboratory.

\section{Chemical analysis}

Samples were analyzed by GC/MS using an Agilent Technologies 7890/5977B instrument. The GC had a splitless injector with helium carrier gas at a constant flow of $1.5 \mathrm{ml}^{\mathrm{min}}{ }^{-1}$. A DBHeavyWax (30 m x $0.25 \mathrm{~mm}$ i.d., $0.25 \mathrm{~mm}$ film thickness; Agilent Technologies) was used, with the column oven programmed from $80^{\circ} \mathrm{C}$ (delay $1 \mathrm{~min}$ ) to $180^{\circ} \mathrm{C}$ at $15^{\circ} \mathrm{C} \cdot \mathrm{min}^{-1}$, then to $190^{\circ} \mathrm{C}$ at $5^{\circ} \mathrm{C} \cdot \mathrm{min}^{-1}$, and finally to 230 ${ }^{\circ} \mathrm{C}$ at $20{ }^{\circ} \mathrm{C} \cdot \mathrm{min}^{-1}$. The MS was operated in the selected ion mode, with the quadrupole and source temperatures set to 150 and $230^{\circ} \mathrm{C}$, respectively. We analyzed only the major component of the pheromone, (Z)-11-hexadecenal (Z1116:Ald), which represents some $90 \%$ by mass of the pheromone (Heath et al. 1991).

For unlabeled compounds, we monitored $m / z 192$ (IS) and 220 (Z11-16:Ald) while, for experiments with ${ }^{13} \mathrm{C}$ labels, we also monitored $\mathrm{m} / \mathrm{z} 222$ and 224 . This set of ions allowed us to determine isotopomers of Z11-16:Ald containing 0 ( $\mathrm{m} / \mathrm{z} 220), 1$ (222) and $2(224){ }^{13} \mathrm{C}_{2}$-acetate monomers for the mass isotopomer distribution analysis (MIDA).

MIDA

This is a combinatorial solution for calculation of precursor enrichment (PE; proportion of a labeled monomer) in a polymer. It has been well discussed (Chinkes 
et al. 1996; Hellerstein and Neese 1992) and we have used it for determining pheromone $P E$ previously (e.g., Foster and Anderson 2011; Foster et al. 2018). Briefly, labeled monomer (in our case ${ }^{13} \mathrm{C}_{2}$-acetate from U-13 $\mathrm{C}$-glucose; $99 \%$ enrichment, from Cambridge Isotopes Ltd, Cambridge, MA) is introduced into a system and the intensities of various isotopomers of a polymer without $[(\mathrm{M}+0)]$ and with labeled monomer are determined. In practice, two labeled isotopomers are monitored, typically those with one $(\mathrm{M}+1)$ and two $(\mathrm{M}+2)$ labeled acetate monomers, allowing calculation of $P E$ of the polymer after allowing for the natural abundances of stable isotopes and the overlap of the spectra of the various isotopomers. We calculated $P E$ using the following equations:

(i) $\operatorname{TTR}(\mathrm{M}+1)=(\mathrm{M}+1 / \mathrm{M}+0)_{\text {post }}-(\mathrm{M}+1 / \mathrm{M}+0)_{\text {pre }}$

(ii) $\operatorname{TTR}(\mathrm{M}+2)=(\mathrm{M}+2 / \mathrm{M}+0)_{\text {post }}-(\mathrm{M}+2 / \mathrm{M}+0)_{\text {pre }}-\mathrm{dT}_{1} \times \mathrm{TTR}(\mathrm{M}+1)$

(iii) $P E=2 \times[\mathrm{TTR}(\mathrm{M}+2) / \mathrm{TTR}(\mathrm{M}+1)] \div[7+\mathrm{TTR}(\mathrm{M}+2) / \mathrm{TTR}(\mathrm{M}+1)]$

The 'pre' and 'post' terms in the tracer to tracee ratio (TTR) indicate intensities of isotopomers before and after $\mathrm{U}^{13} \mathrm{C}$-glucose is introduced. For the pre term, we used theoretical values from known natural abundances. The term $\mathrm{dT}_{1}$ is the contribution of the $(\mathrm{M}+1)$ isotopomer spectrum to the $(\mathrm{M}+2)$ isotopomer intensity.

We used MIDA to calculate the amounts of Z11-16:Ald synthesized before (unlabeled) and after (labeled) U-13C-glucose tracer was fed to females (Foster et al. 2017). Essentially, the calculated $P E$ for a compound in a sample was used to predict the entire isotopomer pattern, with intensities of the isotopomers calculated using the actual $(M+2)$ isotopomer intensity [with spectral overlap from the $(M+0)$ and 
$(M+1)$ isotopomers subtracted]. The isotopomers were summed to calculate (relative to the internal standard) the amount of labeled pheromone. Then, the predicted intensity of the $(\mathrm{M}+0)$ isotopomer in the labeled compound was subtracted from the observed intensity to calculate the amount of unlabeled pheromone. Becuase small intensity values of $(\mathrm{M}+2)$ isotopomers result in large errors in calculation of $p$, we set the minimum threshold for this isotopomer at 200 (well above background intensity in our MS.) Thus, $(M+2)$ areas $<200$ resulted in a calculation of $P E=0$ (i.e., no labeled monomer).

\section{Model simulations}

Our compartmental model for describing the dynamics of the pheromone gland of $C$. virescens has been described in detail (Foster et al. 2018). Briefly, it incorporated all known major processes and state variables from pheromone production to release: a major state variable (titer), an input (synthesis rate), and two outputs (catabolic and release rates). In order to simulate how a higher pheromone release rate affects the amount of pheromone catabolized (question 5, see Introduction), we ran the model for $1 \mathrm{~d}$ females using the new release rate. Since the highest observed release rate (2.17 ng. min $^{-1}$; see Results) we obtained was ca. six times that observed in our original study (ca. 0.33 ng.min ${ }^{-1}$; Foster et al. 2018), we adjusted the release rate constant by a factor of 6 , keeping all other parameters the same. However, this was insufficient to yield the observed release rate, so we adjusted it manually until the observed release rate was obtained using a factor of 10 (to 0.028 ). 


\section{Experiments}

\section{Calling duration and frequency}

We determined the frequency and duration of calling bouts of female $C$. virescens during the scotophase, by recording the behavior of 15 individual virgin $1 \mathrm{~d}$ females using a HC-V130 video camera (Panasonic Corp., Newark, NJ). Fifteen min. prior to the start of the scotophase, a female was placed inside a small arena, consisting of a $2.5 \mathrm{~cm}$ i.d. $x 1.4 \mathrm{~cm}$ high cylinder of translucent nylon tubing, with a clear glass base and top. This kept the female within view and generally hindered her from flipping upside down so that the camera could not record the ovipositor position. The camera was placed under the arena, with a halogen light source with a red gel filter (Times Square Lighting, Stony Point, NY) placed atop and to the side. The light intensity in the arena was regulated to 10 lux, which facilitated detailed viewing of a moth on playback. Study of initial recordings allowed us to categorize a moth's behavior into three states: (1) sedentary, in which the moth remained stationary or moved slowly, with the ovipositor retracted, (2) calling, similar to (1), except the ovipositor was extruded ( $<1$ sec extensions of the ovipositor were not considered), exposing the pheromone gland, and (3) foraging/oviposition, in which the moth moved rapidly around the arena, either with or without its ovipositor extruded; when the ovipositor was extruded, it was dragged over the arena surface with the abdomen arched to facilitate contact of the ovipositor. On playback, we recorded the timing, frequency and duration of each of these categorical behaviors. Occasionally, a moth was in such a position that we could not observe its ovipositor. These 
periods were excluded from the data, reducing the total time (480 min) of observation accordingly.

\section{Pheromone experiments}

In the first series of experiments, we studied native pheromone (i.e., in females that didn't consume labeled glucose) only. As well as validating and optimizing our experimental methods, these experiments addressed questions 2, 3 and part of 4 (see Introduction). In the first experiment, we primarily used the wide funnel and tested the effects of extrusion and time (30 min, $60 \mathrm{~min}$ ) after decapitation in a factorial design. Females were decapitated $1.5-3 \mathrm{~h}$ into the scotophase, and separated into two groups. In one, a clamp was placed across the posterior of the abdomen to extend the ovipositor and evert the gland (simulated calling), while the other was left with the ovipositor and gland retracted (non-calling), with the clip just gripping the lateral edge of the abdomen so as to hold the insect in the collection funnel. The two types of females were then placed immediately into the collection apparatus (Fig. 1). Released pheromone was then collected for either 30 min or $60 \mathrm{~min}$, before the female was removed and the gland analyzed for total pheromone. For comparison, we also included two other treatments: females analyzed for total pheromone immediately after decapitation (i.e., prior to release; $\mathrm{t}$ $=0$ ), and decapitated females with extruded gland in the narrow funnel (Fig. 1). For the latter, pheromone was collected for 30 min and analyzed, and the gland extracted and analyzed for total pheromone. Nine to 13 females were sampled for each treatment. 
The second experiment was similar to the first, except we used the narrow funnel and analyzed glands at the conclusion by rinse/extract sampling. As virtually undetectable amounts of pheromone were detected from retracted glands (see results from first experiment) and there was a possibility of the narrow funnel sealing on the ovipositor and pulling out (extruding) the gland, we only collected pheromone from extruded glands. Thus, the amount collected was compared (by one-way ANOVA) only for the $30 \mathrm{~min}$ and $60 \mathrm{~min}$ extrusions. However, the rinse and gland samplings constituted a factorial design, allowing testing for effects. We also sampled females at $\mathrm{t}=0 \mathrm{~min}$. by the rinse/extract sampling. Nine to 18 females were sampled for each treatment.

For these experiments, factorial designs were analyzed by two-way ANOVA, using amount of Z11-16: Ald as the response and decapitation time and extrusion (extruded or retracted) as independent effects, along with an interaction term. When additional treatments were included, the data were analyzed by one-way ANOVA, with differences in means separated by Student's t-test, with $\alpha$ set at 0.05 .

Since the native pheromone experiments analyzed the fate of pheromone in the gland at time of decapitation, with no differentiation of when it was made (or distributed), we conducted stable isotope labeling experiments that allowed us to address the fate of a newly synthesized pulse of pheromone, and compare its fate over time for non-calling and calling females (questions 4 and 5 in Introduction). 
For this, label was introduced and pheromone biosynthesis allowed for a short period, so that a limited amount of labeled pheromone was produced. In the first pulse experiment, we compared only gland content of females with extruded or retracted glands. Approximately $2 \mathrm{~h}$ into the scotophase, we fed females a $12.5 \mu \mathrm{l}$ drop of $10 \% \mathrm{U}^{13} \mathrm{C}$-glucose solution. Once the female had consumed the entire drop (taking ca. 30-60 sec), it was left for a 10 min pulse of biosynthesis before the female was decapitated. Preliminary experiments showed a good level of pheromone enrichment with a 10 min. pulse. At this point, the female was either left with gland retracted or a vascular clamp placed across the abdomen to evert the gland (simulated calling). For the latter females, the gland was placed immediately inside the narrow funnel with the pump running, although the pheromone collected was not analyzed. The pheromone gland of each of the two types of female was sampled by rinse/extract at various times (decapitation $=$ time 0 ) over the next $90 \mathrm{~min}$. Eight to 10 females were sampled at each time point for each treatment. For the two groups we compared rinse or extract amount of pheromone separately by ANOVA at each time point.

In the second pulse experiment, we collected pheromone for $30 \mathrm{~min}$ from decapitated females with gland extruded using the narrow funnel. Immediately following collection, the gland was sampled by rinse/extract, and the collected (released) pheromone eluted from the DB1 column and also analyzed. Nine females were sampled this way. The amount of pheromone (labeled or unlabeled) was compared across each of the three sampling methods (released, rinse, extract) by a 
mixed regression model with insect identity as a random effect. Means of the three samples for each female were separated by Student's t-test.

\section{RESULTS}

\section{Calling duration and frequency}

Females spent most of their time during the scotophase either in a sedentary state (47\%) or calling (36\%); females exhibited foraging/ovipositional behavior a relatively small percentage (17\%) of the time (Fig 2). Most females called intermittently and frequently through the scotophase. The duration of calling bouts was highly variable, both within and among females, ranging from seconds to hours. Similarly, the frequency of calling among individual females was highly variable with one female not calling at all, while another exhibited 35 distinct bouts.

\section{Pheromone experiments}

In the first experiment, using the wide funnel, we found an effect of extrusion $(p<0.001)$, but not of time $(p=0.871)$, and no interaction $(p=0.867)$ between the two effects, on amount of Z11-16:Ald released (two-way ANOVA with interaction $\left.F_{3,39}=7.68, P<0.001\right)$. Essentially, pheromone was released only when the gland was extruded, but the amount did not differ between 30 min and 60 min collections (Fig. 3a). By contrast, for the residual total pheromone extract, there was no effect of extrusion ( $p=0.711)$, but an effect of time $(p<0.001)$, again with no interaction $\left(p=0.170\right.$ ) between the two effects (two-way ANOVA with interaction $F_{3,39}=4.99, P$ 
$=0.005)$. Essentially, residual gland amount was lower in females after 60 min than after $30 \mathrm{~min}$, regardless of whether the gland was extruded or not (Fig. 3a).

There was a difference (ANOVA, $\mathrm{F}_{2,33}=5.53, \mathrm{p}<0.009$ ) in the amount of pheromone collected between the wide (both at 30 and $60 \mathrm{~min}$ ) and narrow (30 $\mathrm{min}$ ) funnel collections, with more pheromone collected when using the narrow than the wide funnel (Student's t-test, $\mathrm{P}<0.05$; Fig. 3a). Assuming pheromone was released for the entire $30 \mathrm{~min}$ of the collection, the values correspond to rates of $0.77 \mathrm{ng} \cdot \mathrm{min}^{-1}$ (wide funnel) and $2.13 \mathrm{ng} \cdot \mathrm{min}^{-1}$ (narrow funnel), some two and six times, respectively, the rate obtained previously using the glass chamber and Tenax TA adsorbent (Foster et al. 2018). We found an effect (ANOVA, $F_{3,43}=7.25$, $p<0.001$ ) for the corresponding total gland extracts. In this case, the amount of pheromone in the gland before release $(t=0)$ was greater than that for all other treatments (Student's $t$-test, $\mathrm{p}<0.05$ ). The residual amounts in the gland after $30 \mathrm{~min}$ collections (both wide and narrow funnels) were lower than in the 60 min collection (wide funnel only), but only the value for the narrow funnel was different (Fig. 3a).

In the second experiment, using the narrow funnel, we found no difference in the amount of pheromone released between the $30 \mathrm{~min}$ and $60 \mathrm{~min}$ collections (ANOVA $F_{1,28}=0.65, p=0.427 ;$ Fig. 3b). Analysis of the amount of Z11-16:Ald in the rinse showed an effect of both time of collection $(p=0.008)$ and extrusion $(p=0.009)$ with no interaction $(\mathrm{p}=0.350)$ between the terms (two-way ANOVA with interaction, $\left.F_{3,48}=4.89, P=0.005\right)$. Essentially, the amount of Z11-16:Ald in the 
rinse was lower for females with glands extruded than females with glands retracted, with amount also decreasing with increasing time after decapitation, regardless of whether the gland was extruded or retracted. For the residual gland extract, there was an effect of time $(p=0.002)$, but not of extrusion $(p=0.90)$, and no significant interaction $(\mathrm{p}=0.95)$ between the terms (two-way ANOVA with interaction $F_{3,48}=3.82, p=0.016$ ). That is, the amount of Z11-16:Ald in the residual gland extract was similar for females with gland extruded or retracted, with the amount declining for both from 30 to 60 min (Fig. 2b).

The time course of the first pulse experiment showed a distinct difference in amount of labeled Z11-16:Ald between extruded and retracted glands. For both the rinse (Fig. 4a) and residual extract (Fig. 4b), extruded glands showed a rapid and immediate decline before leveling off around $30 \mathrm{~min}$. By contrast, retracted glands showed no decline over the first $30 \mathrm{~min}$., before declining to roughly the same amount as the extruded glands at 90 min. The amounts between the extruded and retracted glands were different at 30 min. for both rinse (ANOVA, $F_{1,24}=10.9, p=$ 0.003 ) and extract (ANOVA, $\left.\mathrm{F}_{1,24}=17.1, \mathrm{p}<0.001\right)$ and at $10 \mathrm{~min}$ for the extract (ANOVA, $\mathrm{F}_{1,24}=6.78, \mathrm{p}=0.016$ ). At the start of the experiment, there was roughly twice as much labeled pheromone in the rinse as in the residual extract, yet near the end of the experiment the two pools had similar amounts.

In the second pulse experiment, roughly $60 \%$ of the labeled Z11-16:Ald accounted for (excluding any catabolized) was released within $30 \mathrm{~min}$., with the balance split 
evenly between the rinse and the residual extract (Fig. 5). The amount in the collection was greater (mixed regression model, Kenward Roger F test $=19.3$, $\mathrm{P}<0.001$; Student's t-test) than the amounts in the rinse and extract. While there were much higher amounts of unlabeled pheromone, they were partitioned similarly to the labeled pheromone among the collection, rinse and gland (Fig. 4). Again, the amount of unlabeled Z11-16:Ald in the collection was greater (mixed regression model, Kenward Roger F test $=20.8, \mathrm{P}<0.001$; Student's t-test) than the amounts in the rinse and extract.

The two pulse experiments allow us to estimate the difference in the amount of pheromone catabolized by simulated simulated calling and non-calling females. If we roughly compare gland content between non-calling and simulated calling females at 30 min ( $1^{\text {st }}$ pulse experiment), they contain (rinse + extract) ca. 26 and 11 ng of labeled pheromone, respectively. Since ca. $20 \mathrm{ng}$ of labeled pheromone was released by simulated calling from $0-30 \mathrm{~min}\left(2^{\text {nd }}\right.$ pulse experiment $)$, then ca. $5 \mathrm{ng}$ more labeled pheromone was catabolized during this period in non-calling than in simulated calling glands.

\section{Model simulations}

The model simulation outputs for a continuously calling female are presented in Figure 6. Of the roughly $600 \mathrm{ng}$ of Z11-16:Ald produced during the scotophase by 1 d females, some 420 ng should be released, representing $70 \%$ (i.e., only $30 \%$ is catabolized). This compares with the ca. $20 \%$ released ( $80 \%$ catabolized) for $1 \mathrm{~d}$ 
females using our previous experimentally determined release rate (Foster et al. 2018). Note, however, the relatively poor fit of the empirical titer data (from (Foster et al. 2018) to that from the model. This is likely a function of the empirical titer data being affected by periods in which the females do not call (thus increasing titer).

\section{DISCUSSION}

Considering the enormous effort over the last 30 years on understanding how female moths biosynthesize precise sex pheromone blends to attract conspecific males (Bjostad et al. 1987; Foster 2016; Jurenka 2003; Löfstedt et al. 2016), we know remarkably little about how pheromone is stored in the gland and how stored pheromone is made available for release. In this study, we developed a combination of techniques to compare the fate of pheromone in simulated calling and non-calling females.

What is the duration and intermittency of calling through the sexually active period (scotophase)?

Female $C$. virescens call intermittently throughout the scotophase, alternating periods of calling with sedentary or foraging/ovipositional behavior. Intermittent or continuous calling throughout the sexually active period have been recorded in other moths (Allison and Cardé 2016b), but it is not known why some species call intermittently while others continuously. Nevertheless, the high degree of intermittency (relatively short and frequent bouts) of calling by females was a little 
surprising, as previously when studying calling periodicity in $C$. virescens through periodic observations, we had observed high levels of calling during each hour of the scotophase (Foster et al. 2018). It is possible that this high degree of intermittency is a characteristic of our colony, which has been in laboratory culture for a large number of generations, or it may be a result of the small arenas used. Nevertheless, given the high release rates of pheromone we obtained in this study (see below), this intermittency in calling may be a behavioral mechanism to allow gland storage levels to refill, enabling females to maintain a high rate of release when they call again.

Do females release pheromone at a higher rate than originally determined and, if so, how does this affect the amount catabolized?

We previously estimated $80 \%$ of pheromone produced by $1 \mathrm{~d}$ females was catabolized throughout the scotophase (Foster et al. 2018). However, using the substantially higher empirical release rate obtained in this study, our model simulation showed that only $30 \%$ of pheromone produced by a continuously calling female is catabolized. While this shows a striking reversal, with most pheromone now being released, we caution that this percentage should be considered as indicative rather than absolute. First, the model assumes that females call continuously, which contrasts with our behavioral observations and poor correspondence of titer with empirical data. Thus, the percentage catabolized will be modulated by the actual percentage of time a female calls. Second, the release rate from a decapitated female may be different from that of a freely calling female. 
Our collection time was $30 \mathrm{~min}$, by which all available pheromone had been collected. Using a different collection method with forced gland extrusion, Pope et al. (1982) found that all pheromone was collected within $10 \mathrm{~min}$ from female $C$. virescens or H. zea. If all the pheromone had been collected in a shorter time than 30 min, our calculated release rate would be proportionally higher. Furthermore, decapitated females had stopped producing pheromone. Intact females continue to produce pheromone and may release even greater amounts within the same time period. However, it is also possible that our collection setup may have yielded a higher release rate than that by intact females in nature. Evaporation rates of semivolatile chemicals are influenced by air speed (Mackay and van Wesenbeeck 2014). In our setup, the close fit of the funnels around the gland or abdomen could have resulted in high airspeeds over the gland, effectively 'stripping" pheromone at an enhanced rate compared to that in nature. This might explain why we collected more pheromone with the narrow, than with the wide, funnel. Unfortunately, we could not measure localized airspeeds in our apparatus. Collecting in the vicinity of freely calling intact females, as in Nojima et al. (2018), should be used to determine actual release rates.

Is calling behavior an effective on/off method for pheromone release? While this has typically been assumed (e.g., Nojima et al. 2018), it has not, to our knowledge, been rigorously tested for individual females. Forced extrusion of the gland (simulated calling) resulted in pheromone being released at a high rate, while gland retraction resulted in little or no pheromone detection. Thus, gland 
extrusion/retraction is a highly effective on/off mechanism for controlling pheromone release in $C$. virescens and probably other moths. The surface of the pheromone gland of the closely related noctuid moth Helicoverpa zea has many convolutions that are thought to increase surface area and aid evaporation of pheromone when the ovipositor is extended and the gland everted (Raina et al. 2000). When the ovipositor and gland are retracted, the converse likely occurs, with the gland and adjacent cuticle involuting, effectively "sealing off" the cuticular surface from airflow and preventing release of the semivolatile pheromone to the environment.

How does storage and catabolism of pheromone differ between calling and non-calling females?

At the start, most (ca. 70\%) pheromone was found in the rinse (i.e., on or near the surface of the gland cuticle) fraction, with a smaller amount in the extract (deeper in the cuticle). Over time, pheromone in the gland declined in both non-calling and simulated calling females, although the decline was more rapid in the latter due to release. Pheromone that was released appears to have come largely from the rinse pool, as indicated by the change in rinse to extract ratio from ca. 2:1 at 0 min to ca. $1: 2$ at $30 \mathrm{~min}$. While non-calling females also stored most pheromone in the same part of the gland, the lack of release meant that the quantity declined less. This is evident by the ratio of rinse to extract in non-calling females being ca. 1.2:1 after 30 min. Both simulated calling and non-calling females showed a decline in pheromone in both rinse and extract due to catabolism, although more pheromone was 
catabolized in non-calling than in simulated calling females. Thus, not calling may allow pheromone storage levels to increase, but at the cost of catabolizing more pheromone.

Is there a relatively high pheromone flux from production to release?

The pulse experiments showed that, not only is the pulse of labeled pheromone produced very quickly after PBAN stimulation (as shown previously, Foster and Anderson 2018), but a high percentage of it is translocated rapidly (i.e., a high flux) to the surface for release. Within 40 minutes of feeding (i.e., at 30 min in the experiments) on labeled glucose, $>60 \%$ of the labeled pheromone accounted for (excluding any catabolized) had been released, with the balance, in the gland, split evenly between the rinse and extract pools. Interestingly, the ratio of labeled pheromone across the three pools (released, rinse and residual extract) was similar to that for unlabeled pheromone; i.e., 'newer' pheromone was partitioned across the three pools similar to that of 'older' pheromone. Although we only sampled pheromone 30 min after decapitation (shorter times might reveal different distributions), this suggests that newly synthesized pheromone or precursor is not immediately translocated to the surface for release but is, rather, mixed with a residual pool of older material prior to translocation. Since the pheromone precursor (Z)-11-hexadecenol (Z11-16:0H) is thought to be synthesized and stored in gland cells (Foster and Anderson 2019; Hagström et al. 2013), a likely scenario is that newly synthesized Z11-16:0H is mixed with 'older' Z11-16:0H in gland cells, from where it is translocated to the cuticle and oxidized to (mixed 'old' and 'new') 
Z11-16:Ald (Teal and Tumlinson 1986), before being translocated to the cuticular surface for release.

In summary, not only is the gland of female $C$. virescens considerably more efficient, in terms of the proportion of pheromone released Vs. catabolized, than previously thought, but it is also effective at rapidly shutting on/off a high flux of pheromone for release.

\section{Acknowledgments}

Funding for this work was provided by United States Department of Agriculture Hatch Project ND02388. We also thank the United States Department of Agriculture-National Institute of Food and Agriculture for an Instrument Grant, 2015-07238 contributing, in part, to the purchase of the GC/MS system. 


\section{References Cited}

Allison JD, Cardé RT (eds) (2016a) Pheromone communication in moths: evolution, behavior and application. University of Caifornia Press, Oakland, California

Allison JD, Cardé RT (2016b) Variation in moth pheromone: causes and consequences. In: Allison JD, Cardé RT (eds) Pheromone communication in moths: evolution, behavior and application. University of California Press, Oakland, California, pp 25-41

Almeida ÂA, Lima ER, Reis Jr R (2008) Pupal Period Affects Calling Behavior of the Wheat Moth, Pseudaletia sequax (Lepidoptera: Noctuidae) Ethology 114:499503 doi:10.1111/j.1439-0310.2008.01492.x

Bendib A, Minet J (1998) Female pheromone glands in arctiidae (Lepidoptera). Evolution and phylogenetic significance Comptes Rendus de l'Académie des Sciences - Series III - Sciences de la Vie 321:1007-1014 doi:https://doi.org/10.1016/S0764-4469(99)80056-0

Bjostad LB, Wolf WA, Roelofs WL (1987) Pheromone biosynthesis in lepidopterans: Desaturation and chain shortening. In: Prestwich GD, Blomquist GJ (eds) Pheromone Biochemistry. Academic Press, New York, pp 77-120

Chinkes DL, Aarsland A, Rosenblatt J, Wolfe RR (1996) Comparison of mass isotopomer dilution methods used to compute VLDL production in vivo American Journal of Physiology - Endocrinology and Metabolism 271:E373383

Eltahlawy H, Buckner JS, Foster SP (2007) Evidence for two-step regulation of pheromone biosynthesis by the pheromone biosynthesis-activating neuropeptide in the moth Heliothis virescens Arch Insect Biochem Physiol 64:120-130

Foster S, Anderson K (2011) The use of mass isotopomer distribution analysis to quantify synthetic rates of sex pheromone in the moth Heliothis virescens. J Chem Ecol 37:1208-1210

Foster SP (1993) Neural inactivation of sex pheromone production in mated lightbrown apple moths, Epiphyas postvittana (Walker). J Insect Physiol 39:267-273 doi:doi:10.1016/0022-1910(93)90098-C

Foster SP (2016) Toward a Quantitative Paradigm for Sex Pheromone Production in Moths. In: Allison JD, Cardé RT (eds) Pheromone Communication in Moths: Evolution, Behavior, and Application. University of California Press, Oakland, CA, pp 113-126

Foster SP, Anderson KG (2018) Differential Pheromone Sampling of the Gland of Female Heliothis virescens Moths Reveals Glandular Differences in Composition and Quantity J Chem Ecol 44:452-462 doi:10.1007/s10886018-0954-0

Foster SP, Anderson KG (2019) Production and Distribution of Aldehyde and Alcohol Sex Pheromone Components in the Pheromone Gland of Females of the Moth Chloridea virescens J Chem Ecol 45:9-17 doi:10.1007/s10886-0181041-2

Foster SP, Anderson KG, Casas J (2017) Sex pheromone in the moth Heliothis virescens is produced as a mixture of two pools: de novo and via precursor 
storage in glycerolipids Insect Biochemistry and Molecular Biology 87:26-34 doi:http://dx.doi.org/10.1016/j.ibmb.2017.06.004

Foster SP, Anderson KG, Casas J (2018) The Dynamics of Pheromone Gland Synthesis and Release: a Paradigm Shift for Understanding Sex Pheromone Quantity in Female Moths J Chem Ecol 44:525-533 doi:10.1007/s10886-0180963-z

Groot AT (2014) Circadian rhythms of sexual activities in moths: a review Frontiers in Ecology and Evolution 2 doi:10.3389/fevo.2014.00043

Hagström ÅK, Walther A, Wendland J, Löfstedt C (2013) Subcellular localization of the fatty acyl reductase involved in pheromone biosynthesis in the tobacco budworm, Heliothis virescens (Noctuidae: Lepidoptera) Insect Biochemistry and Molecular Biology 43:510-521 doi:http://dx.doi.org/10.1016/j.ibmb.2013.03.006

Heath RR, McLaughlin JR, Proshold F, Teal PEA (1991) Periodicity of female sex pheromone titer and release in Heliothis subflexa and H. virescens (Lepidoptera: Noctuidae) Ann Entomol Soc Am 84:182-189

Hellerstein MK, Neese RA (1992) Mass isotopomer distribution analysis: a technique for measuring biosynthesis and turnover of polymers American Journal of Physiology - Endocrinology And Metabolism 263:E988-1001

Johansson B, Jones T (2007) The role of chemical communication in mate choice Biol Rev Camb Philos Soc 82:265-289 doi:DOI: 10.1111/j.1469185X.2007.00009.x

Jurenka R (2017) Regulation of pheromone biosynthesis in moths Current Opinion in Insect Science 24:29-35 doi:https://doi.org/10.1016/j.cois.2017.09.002

Jurenka RA (2003) Biochemistry of female moth sex pheromones. In: Blomquist GJ, Vogt RG (eds) Insect Pheromone Biochemistry and Molecular Biology. Elsevier Academic Press, London, UK, pp 53-80

Löfstedt C, Wahlberg N, Millar JG (2016) Evolutionary patterns of pheromone diversity in Lepidoptera. In: Allison JD, Cardé RT (eds) Pheromone communication in moths: evolution, behavior and application. University of California Press, Oakland, California, pp 43-78

Ma PWK, Ramaswamy SB (2003) Biology and ultrastructure of sex pheromoneproducing tissue. In: Blomquist GJ, Vogt RC (eds) Insect pheromone biochemsitry and molecular biology. Elsevier Academic Press., London, pp 19-51

Mackay D, van Wesenbeeck I (2014) Correlation of Chemical Evaporation Rate with Vapor Pressure Environmental Science \& Technology 48:10259-10263 doi:10.1021/es5029074

Nojima S, Classen A, Groot AT, Schal C (2018) Qualitative and quantitative analysis of chemicals emitted from the pheromone gland of individual Heliothis subflexa females PLOS ONE 13:e0202035 doi:10.1371/journal.pone.0202035

Pope MM, Gaston LK, Baker TC (1982) Composition, quantification, and periodicity of sex pheromone gland volatiles from individual Heliothis virescens females J Chem Ecol 8:1043-1055 doi:10.1007/bf00987885 
Raina AK, Wergin WP, Murphy CA, Erbe EF (2000) Structural organization of the sex pheromone gland in Helicoverpa zea in relation to pheromone production and release Arthropod Structure and Development 29:343-353

Teal PEA, Tumlinson JH (1986) Terminal steps in pheromone biosynthesis by Heliothis virescens and $H$. zea J Chem Ecol 12:353-366

Turgeon JJ, McNeil JN (1983) Modifications in the calling behaviour of Pseudaletia unipuncta (Lepidoptera: Noctuidae), induced by temperature conditions during pupal and adult development. The Canadian Entomologist 115:10151022 doi:10.4039/Ent1151015-8 
Figure legends

Figure 1. The pheromone collection apparatus illustrating how the pheromone gland of decapitated Chloridea virescens was extruded by a vascular clamp and inserted into the wide (top) and narrow (bottom) funnels.

Figure 2. Behaviors of virgin Chloridea virescens females (1 d) during the scotophase $(8 \mathrm{~h})$. Means and ranges (time and frequency) are of all events observed across 15 individual females. The pie represents the mean percentage of time for each behavior by the 15 females.

Figure 3. Mean amounts of (Z)-11-hexadecenal from decapitated Chloridea virescens females. (a) Released and whole gland extracts (taken after collection) at time of decapitation $(\mathrm{t}=0)$, with gland forcibly extruded and pheromone collected via a wide funnel for 30 (W30) or $60 \mathrm{~min}$ (W60), with gland retracted and pheromone collected via a wide funnel for 30 (W30-R) or $60 \mathrm{~min}$ (W60-R), and with gland forcibly extruded and pheromone collected via a narrow funnel for 30 min (N30). (b) Released, rinse and extract samplings with gland forcibly extruded and pheromone collected via a narrow funnel for 30 (N30) or 60 (N60) min, and gland retracted and sampled (extract) after 30 (N30-R) or 60 min (N30-R). Note, when the gland was retracted, pheromone was not collected. SEMs are atop bars. In (a) bars with different letters (of the same case) have means that are different (Students t-test, $\mathrm{p}<0.05$ ). In (b), NS indicates no difference (ANOVA, $\mathrm{p}<0.05$ ) between the amounts collected in N30 and N60 treatments (comparison marked by parenthesis).

Figure 4. Mean amounts of labeled (Z)-11-hexadecenal (Z11-16:Ald) over time (after decapitation) in first stable isotope pulse experiment. Females were fed $\mathrm{U}-{ }^{13} \mathrm{C}-$ glucose and a $10 \mathrm{~min}$. pulse of pheromone biosynthesis allowed before females were decapitated, and the pheromone gland either forcibly extruded (E) or left retracted (R). Glands were then sampled by rinse (a) and extract (b) over time. SEMs are given. An asterisk indicates mean amounts of Z11-16:Ald in R and E females were different (ANOVA, $\mathrm{p}<0.05$ ) at a given time.

Figure 5. Mean amounts of labeled and unlabeled $(Z)$-11-hexadecenal in the second stable isotope pulse experiment. Females were fed U-13C-glucose and a $10 \mathrm{~min}$. pulse of pheromone biosynthesis allowed before females were decapitated and the pheromone gland forcibly extruded. Pheromone was collected (release) for $30 \mathrm{~min}$ before the gland was sampled by the rinse and extract approach. SEMs are given. Bars with different letters (of the same case) have means that are different (mixed regression model, Students t-test, $\mathrm{p}<0.05$ ).

Figure 6. Compartmental model simulations, based on the higher release rate determined in this study, for amount of $(Z)$-11-hexadecenal synthesized, stored (titre), degraded and released for $1 \mathrm{~d}$-old female Chloridea virescens over the course 
of a scotophase. The open circles in the titre plot are empirical data (from Foster et al. 2018; see also for detailed model description). 


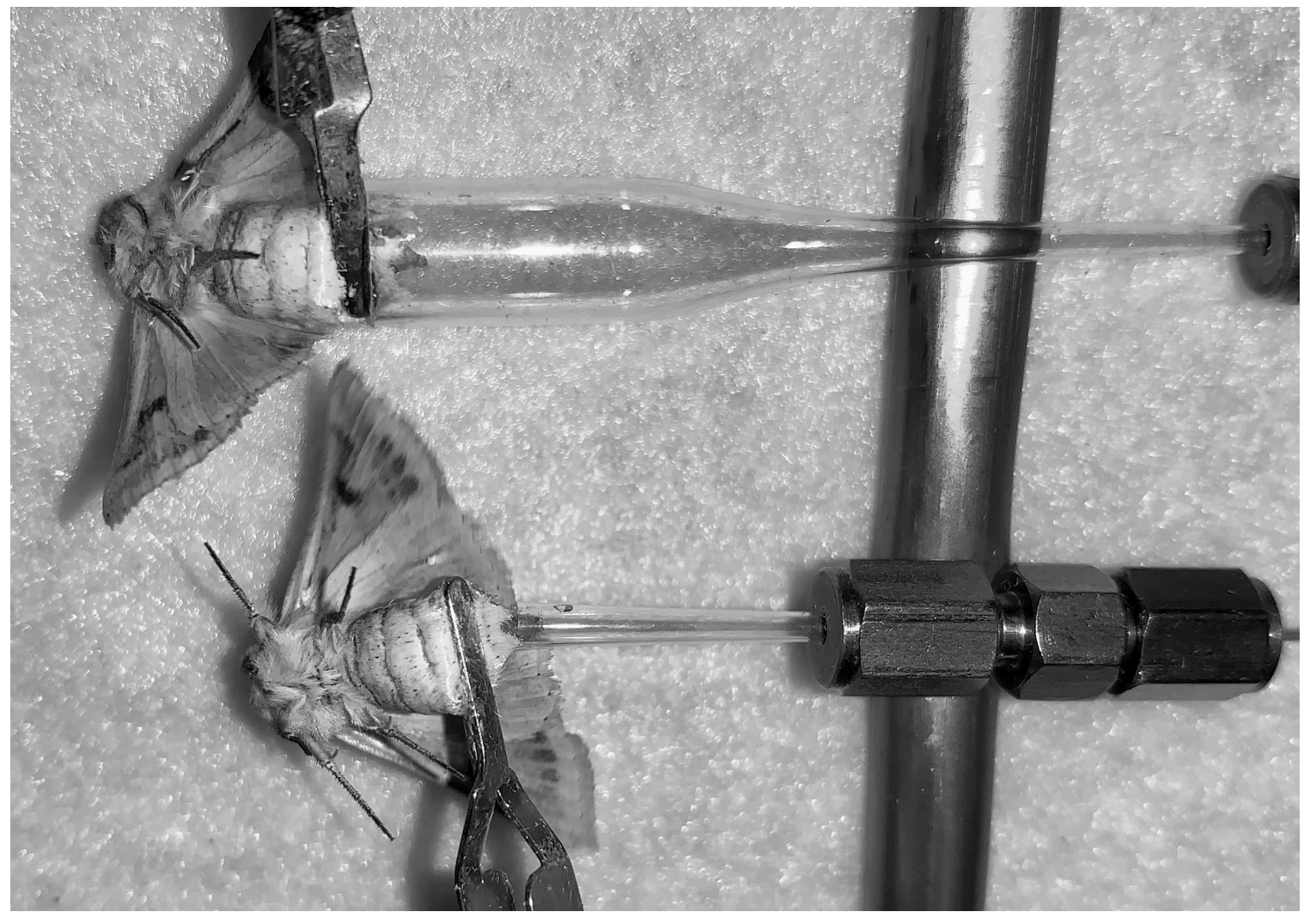




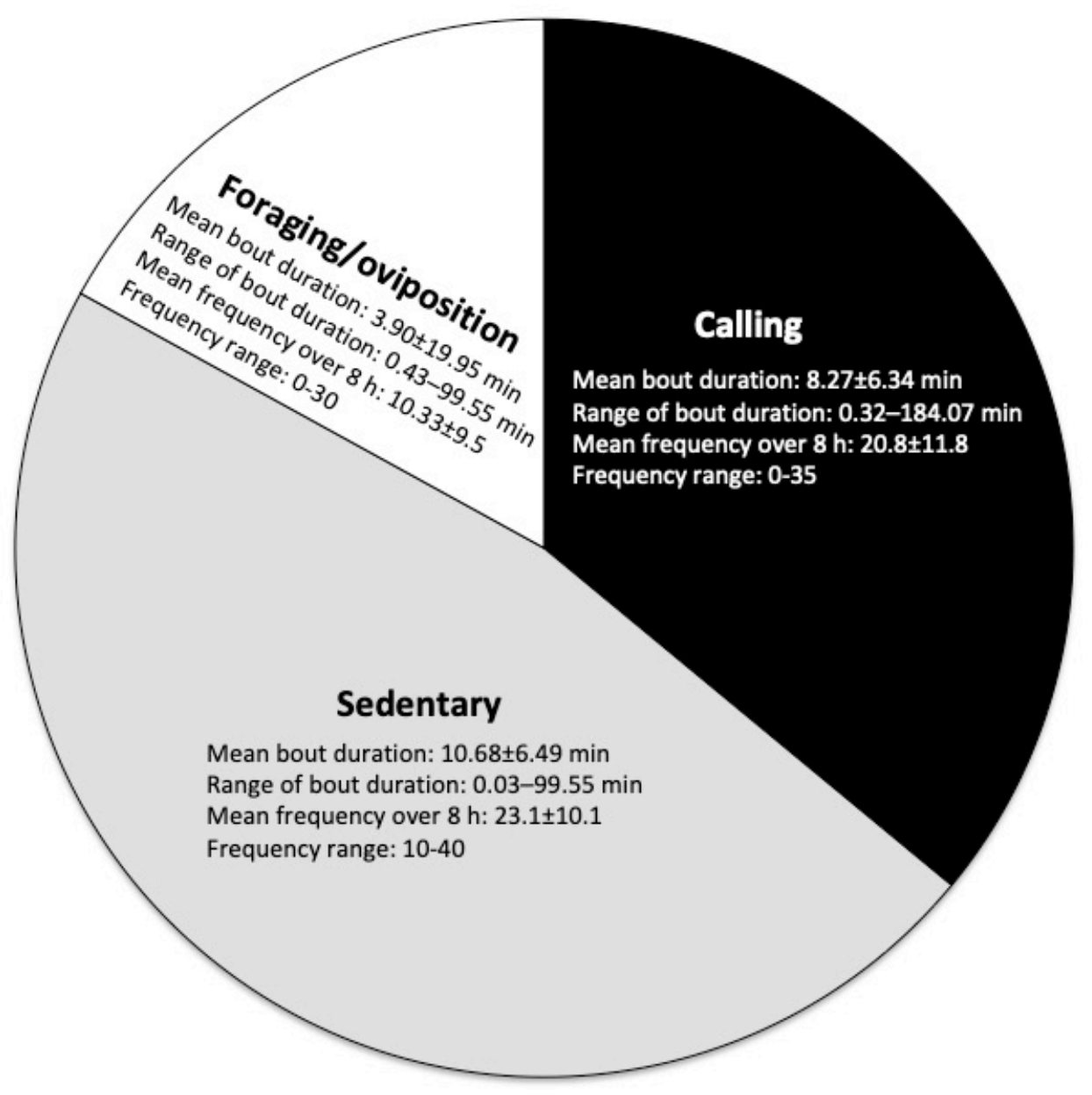

Figure 2 
a)

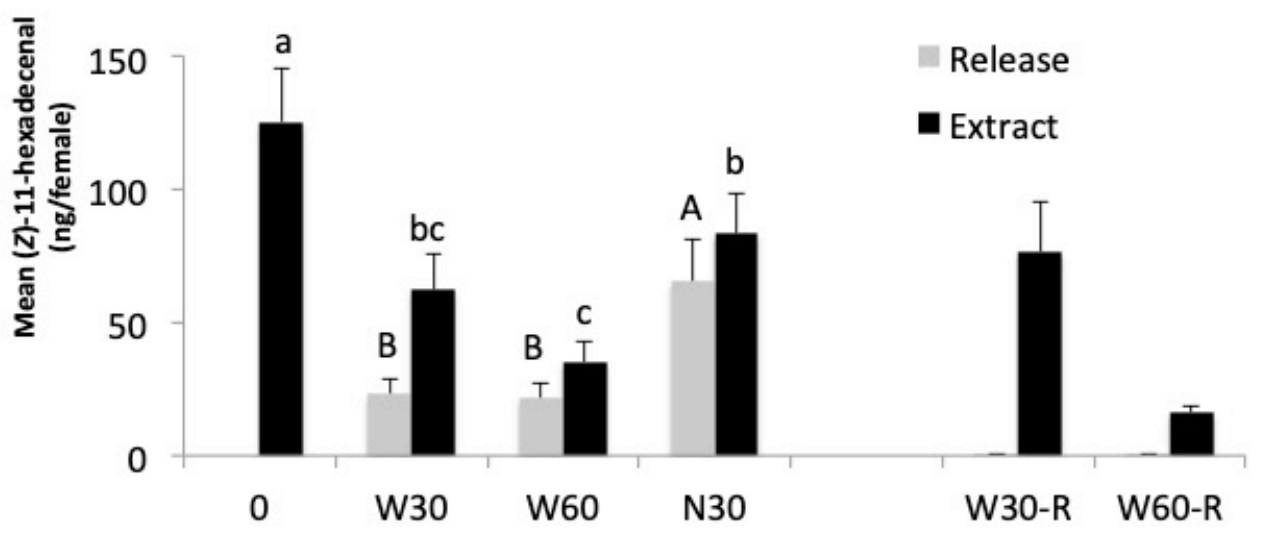

b)

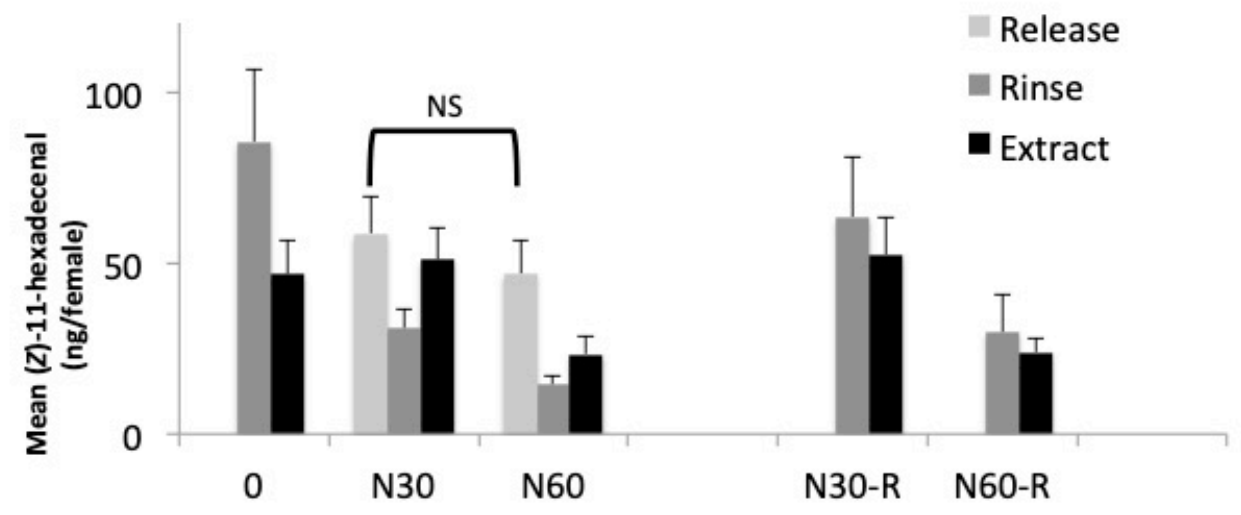

Fig. 3 

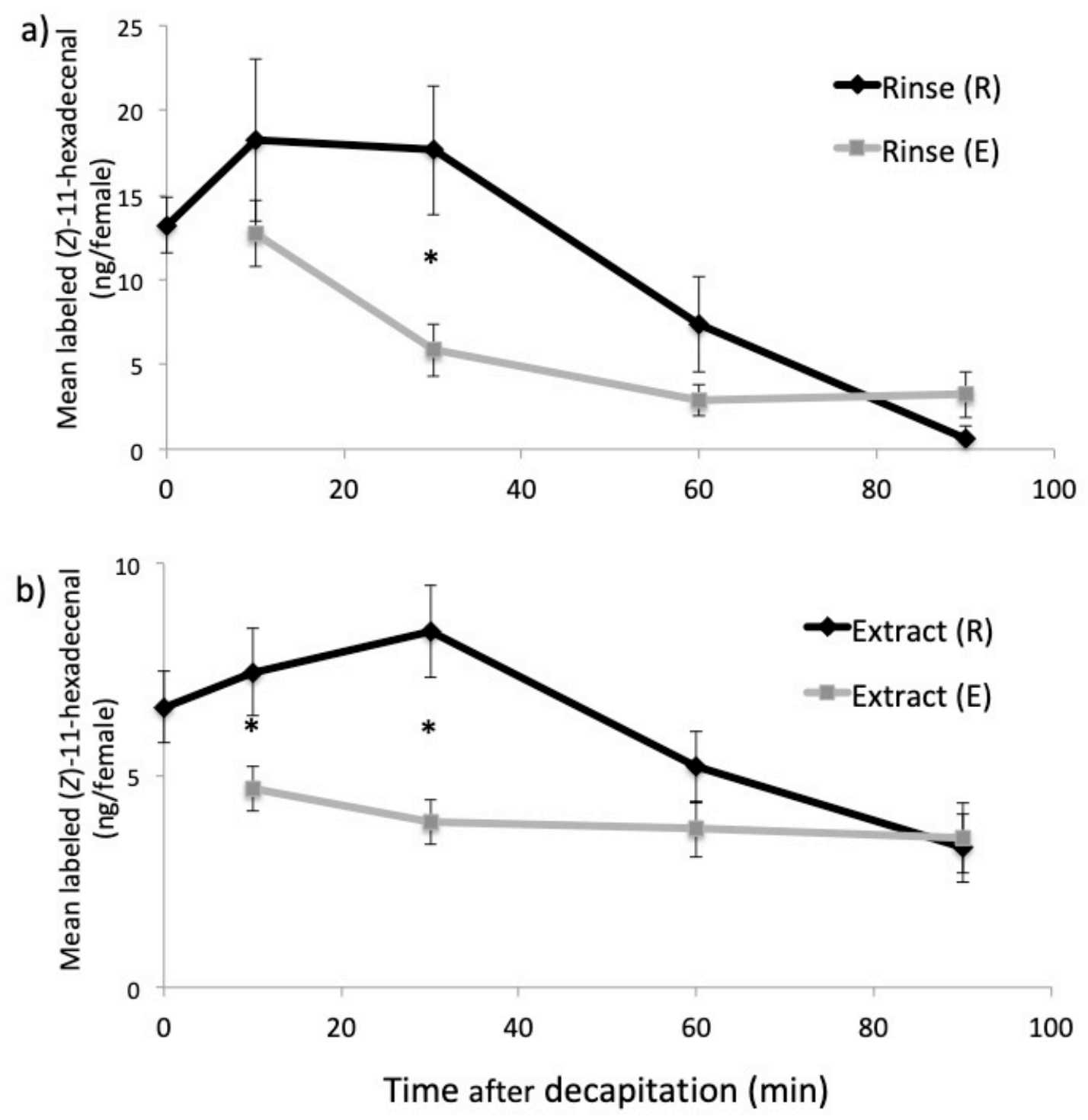

Fig. 4 


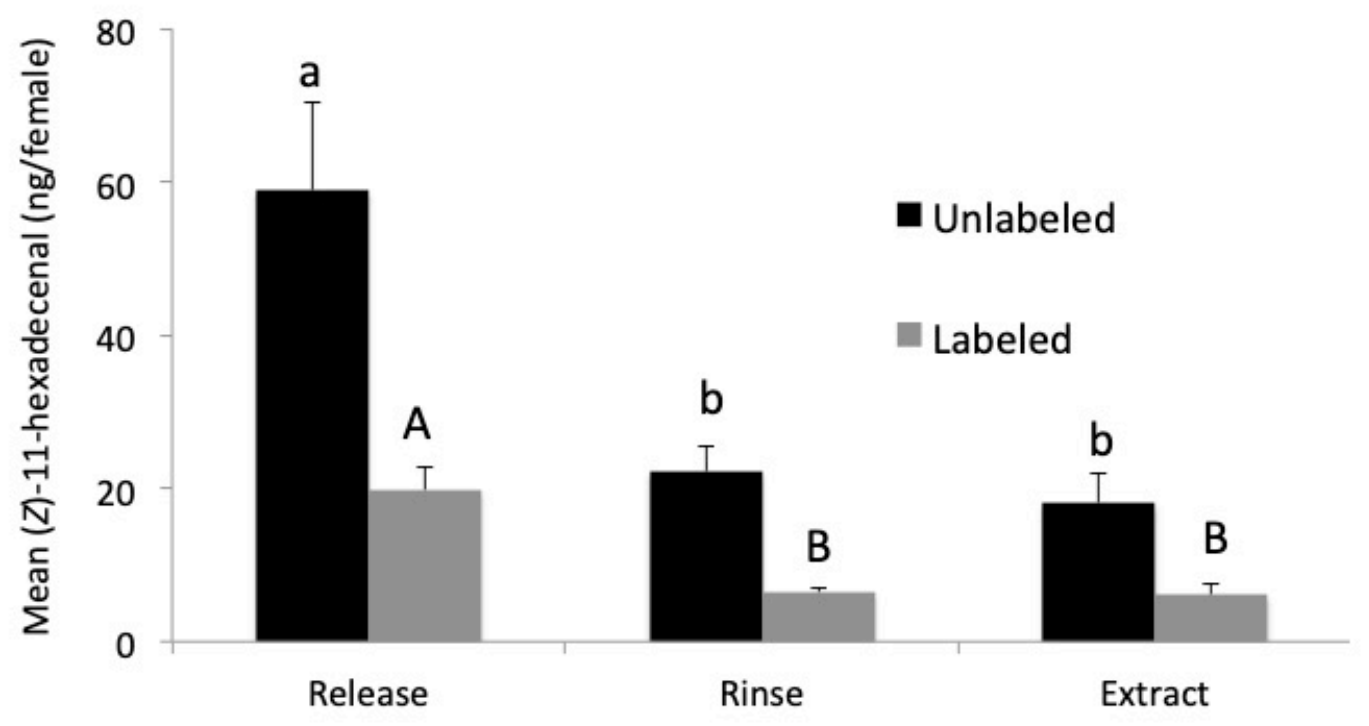

Fig. 5 

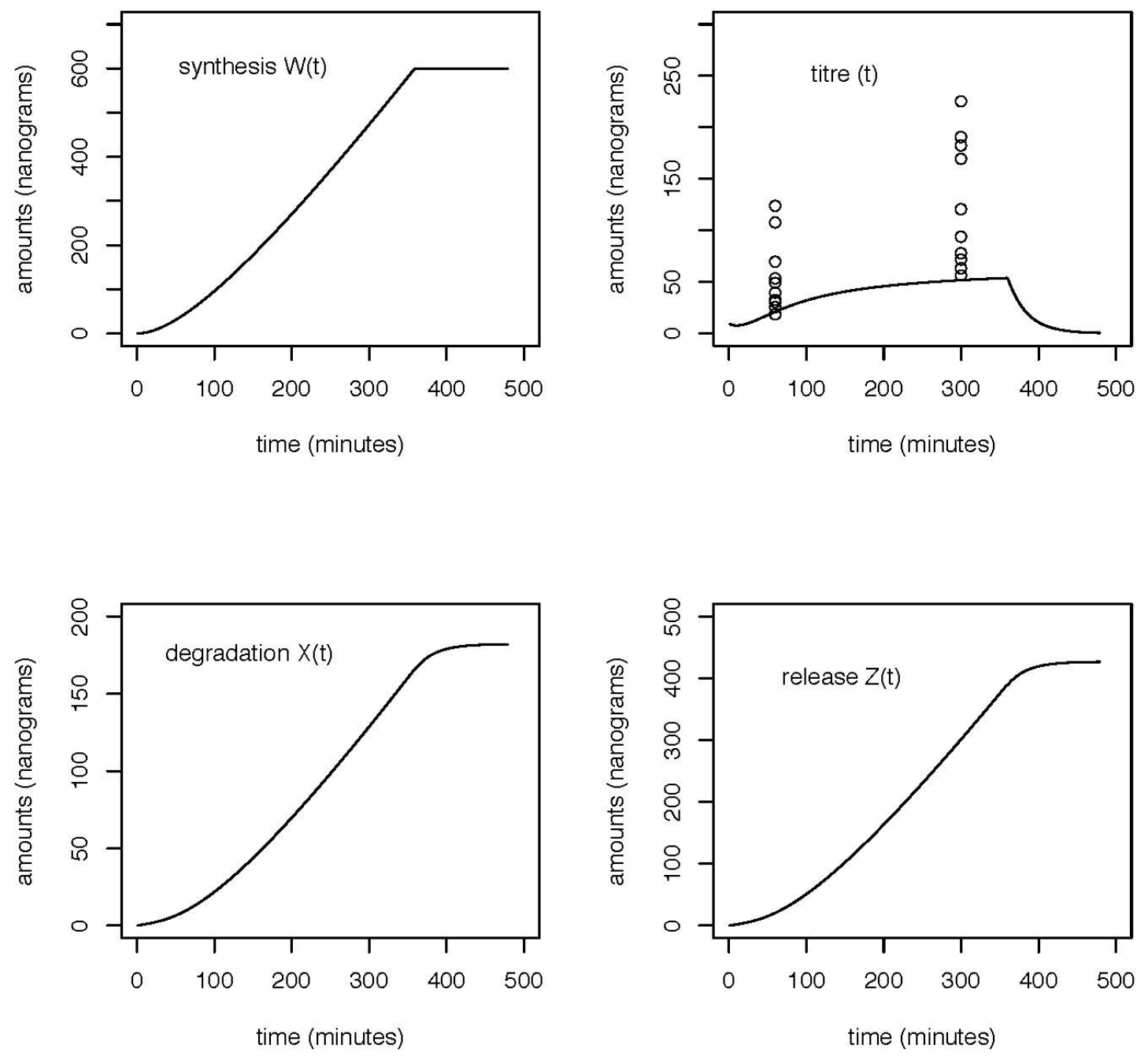

Fig. 6 